\title{
Achieving Connectivity through Coalescence in Mobile Robot Networks
}

\author{
Sameera Poduri ${ }^{1}$ and Gaurav S. Sukhatme $e^{1,2}$ \\ ${ }^{1}$ Department of Computer Science \\ 2 Department of Electrical Engineering \\ University of Southern California \\ Los Angeles, CA-90089 \\ Email: sameera, gaurav@usc.edu
}

\begin{abstract}
Coalescence is the problem of isolated mobile robots independently searching for peers with the goal of forming a single connected network. This paper analyzes coalescence time for a worst-case scenario where the robots do not have any knowledge about the environment or positions of other robots and perform independent, memoryless search. Using the random direction mobility model, we show that coalescence time has an exponential distribution which is a function of the number of robots, speed, communication range, and size of the domain. Further, as the number of robots $(N)$ increases, coalescence time decreases as $O\left(\frac{1}{\sqrt{N}}\right)$ and $\Omega\left(\frac{\log (N)}{N}\right)$. Simulations validate our analysis and also suggest that the lower bound is tight. This paper is an extension of [1] where we studied a simplified setting with a stationary base station that the robots search for and coalesce to.
\end{abstract}

\section{INTRODUCTION}

Communication connectivity is both essential and challenging for networked mobile robots. While on one hand robot groups rely on network-wide connectivity for coordination, on the other hand maintaining connectivity in a large group of moving robots is hard because connectivity is an inherently global property. Distributed algorithms to maintain connectivity constrain the motion of robots and are often expensive in terms of computation and communication. Another difficulty is that the communication range for most low power radios is highly irregular [2] which adds to the unpredictability of connectivity. Given the high cost of maintaining connectivity, we are interested in the question - what happens if connectivity is lost? This situation could arise due to several reasons robots could start in a disconnected state, or accidentally lose connectivity due to failures, or intentionally disconnect for efficient task completion. The cost of regaining connectivity, in terms of time and energy spent, should be taken into account while designing the motion coordination algorithm. If the cost is high the coordination algorithm should minimize the chances of disconnections and if the cost is low it should allow the robots to occasionally disconnect and rejoin the network after task completion.

This work is supported in part by the National Science Foundation (NSF) under grants CNS-0325875, IIS-0133947, CNS-0540420, CNS-0520305 and grants CCR-0120778, ANI-00331481 (via subcontract). We also gratefully acknowledge support from the Okawa foundation, and USC Women in Science and Engineering (WiSE) program.
Coalescence is the problem of isolated mobile robots independently searching for peers with the goal of forming a single connected network. In this paper, we study the time taken for coalescence. We consider the scenario where the robots are memoryless (do not build a map) and do not have any knowledge of the environment or positions of the other robots. Each robot performs an independent random search and when two robots meet, they coalesce into a cluster and the cluster thereafter sticks together and moves as a single random walk. This is illustrated in figure 1. While the memoryless model is realistic for large swarms of simple robots, in several applications robots will be capable of performing systematic exploration and mapping to speed up coalescence. The coalescence time in this case is tied to the exploration algorithm and structure of the domain and the memoryless random walk provides a simplified worst case study.

While coalescence can be considered a variation of the rendezvous problem, we use the term coalescence to emphasize that the communication spread of the clusters increases as more robots join. As we will show in section IV-C, the communication spread plays an important role in determining latency. The basic idea is that robots need not be collocated to remain connected. If they remain spread out, the disconnected robots will have a better chance of discovering them. The coalescence time can be further improved if the clusters form an optimal shape such as a line and move in a direction that maximizes the sweep area. This will involve a significant coordination overhead because maintaining a formation while moving through an environment with obstacles is challenging [3]. Therefore we do not make any assumption on the shape of the cluster.

The outline of the paper is as follows. Section II discusses related work. Section III presents the problem formulation and mobility model. In section IV we derive the coalescence time based on the meeting time of the mobility model. The coalescence time has an exponential distribution which is a function of the number of robots, speed, communication range, and size of the domain. The main challenge in the analysis is that the time between two clusters hitting each other depends on the size and shape of the clusters which are both random and difficult to model. We make several simplifying 


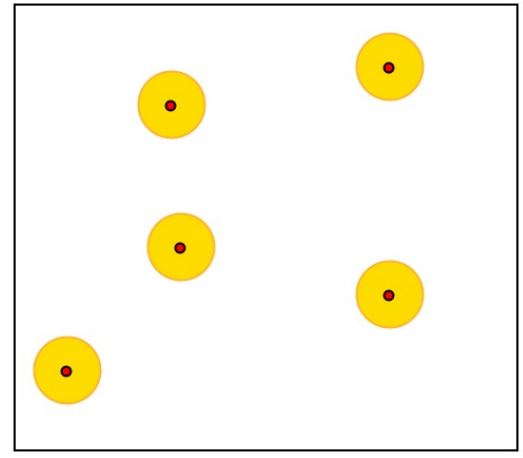

(a) $t=0$

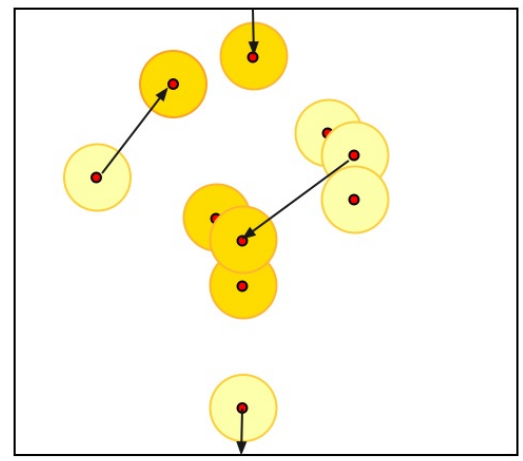

(d) $t=M T_{2}^{5}<t<M T_{3}^{5}$

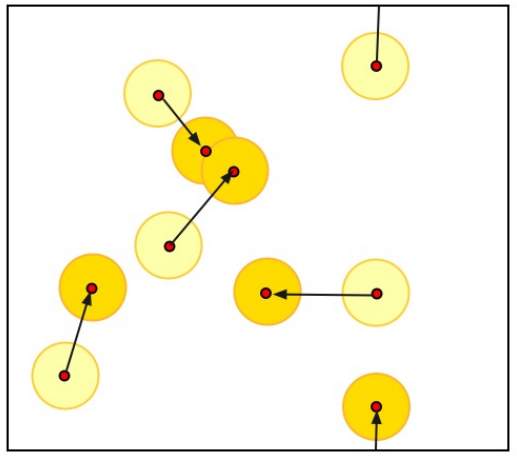

(b) $t=M T_{1}^{5}$

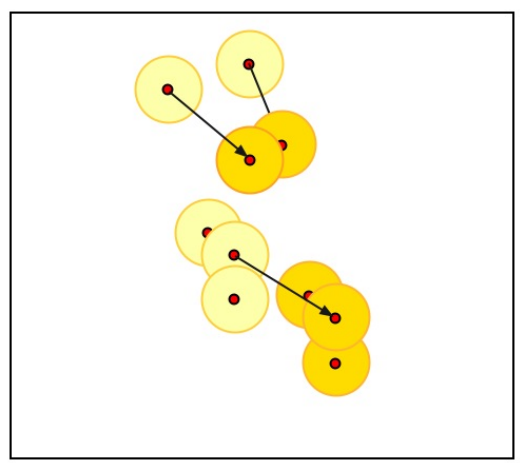

(e) $t=M T_{3}^{5}$

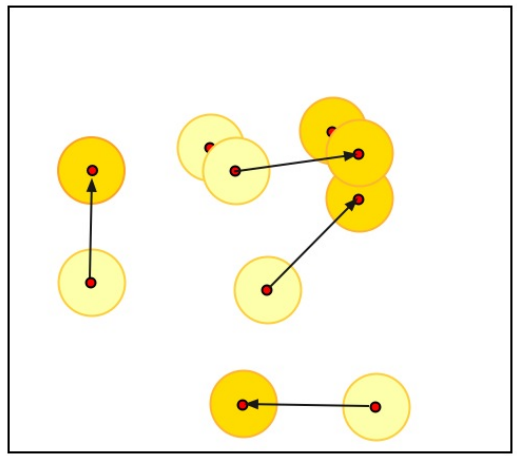

(c) $M T_{2}^{5}$

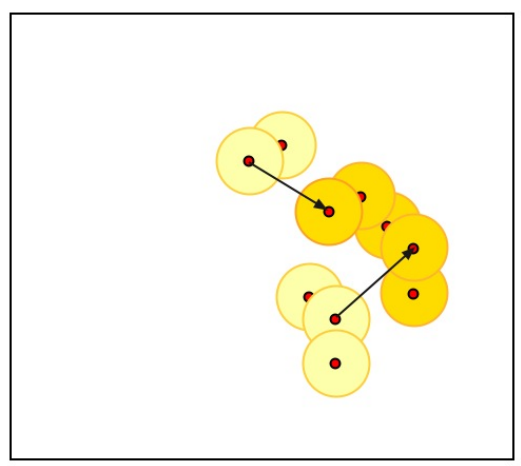

(f) $t=M T_{4}^{5}=C T(N=5)$

Fig. 1. Illustration of Coalescence for $N=5$. The dark red circles represent robots and the yellow discs around them are their communication regions. The arrows show the random walk steps with the faded discs being the robot positions at the previous time instant. As robots coalesce, the communication spread of clusters grows and the robots that are still searching have a better chance of hitting it.

assumptions to make the analysis tractable which are validated using simulations in section V. We are particularly interested in the asymptotic behavior of the coalescence time with respect to the size of the network, $N$. In section IV-C we consider three extreme scenarios and show that coalescence time is $O\left(\frac{1}{\sqrt{N}}\right)$ and $\Omega\left(\frac{\log (N)}{N}\right)$. One of these scenarios is based on our earlier work [1] with a stationary base station where robots coalesce only upon hitting the base station or the cluster containing the base station. Simulations in MATLAB (section $\mathrm{V}$ ) verify these bounds and also suggest that the lower bound is tight i.e. the coalescence time is $\Theta(\log (N) / N)$.

\section{RELATED WORK}

Rendezvous is the problem of multiple mobile robots meeting at a point in an unknown environment. Several distributed algorithms have been proposed. Some of these assume connectivity [4], [5]. If the communication graph is a Relative Neighborhood Graph then rendezvous algorithms have been proposed that can deal with partial communication loss over some of the links [6]. In the absence of connectivity, the robots can explore the environment while building a map of the landmarks [7] where other robots are likely to visit. In coalescence, the main objective is to attain communication connectivity as against colocation.

Several algorithms have been proposed to maintain connectivity of a wireless network [8], [9] given that the initial network is connected. Coalescence algorithms complement these by providing a way to regain connectivity in the event of failures. In [10], the variation in connectivity of a network of robots performing random walks on a lattice has been analyzed. Recently, the role of mobility in increasing sensing coverage of a network has been analyzed [11]. In particular, it has been shown that the time taken to detect an intruder decreases in comparison to a static network. In delay tolerant networks, mobile nodes are disconnected most of the time and communicate when they are within communication range of each other. To study the routing delay, the motion of the mobile nodes is modeled as a random mobility model. In both the above cases, achieving connectivity is not the goal and the nodes continue moving after an intruder is detected or message is exchanged.

In random walks literature, a coalescing random walk refers to a system of particles that coalesce when they hit other particles while performing a random walk [12]. Several asymptotic properties such as the time for convergence have been analyzed. Here, a group of coalesced particles is equivalent to one particle and this analysis does not capture the increase in communication spread.

\section{Problem Statement and Models}

We are interested in the following problem. 


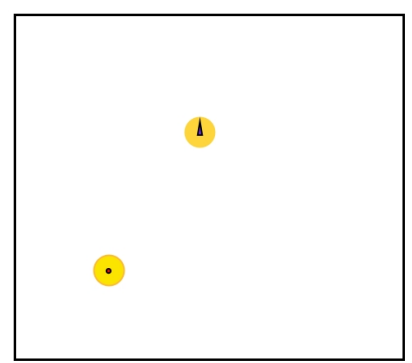

(a) $t=0$

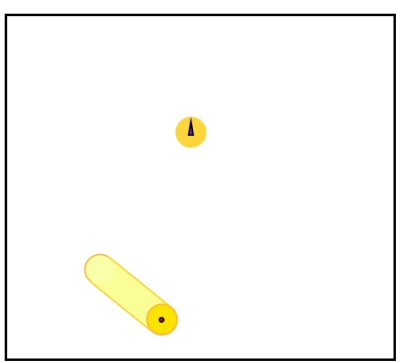

(b) $t=1$

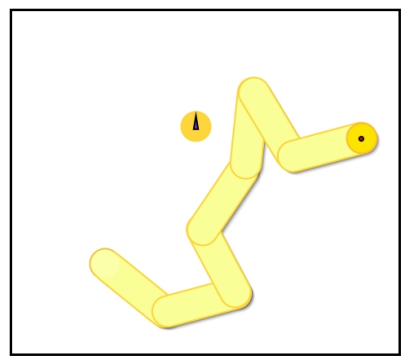

(c) $t=7$

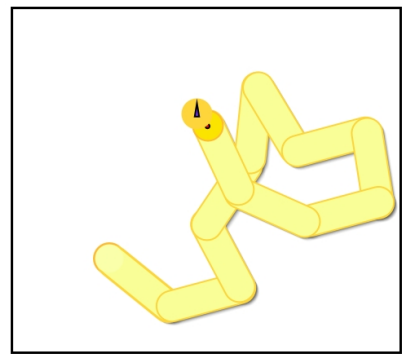

(d) $H T=t=11$

Fig. 2. Illustration of random direction mobility model, $R M$. The blue cone and dark red circle are a stationary node and the isolated robot respectively along with their communication discs. The light yellow region is the area that the robot has "covered" in its search for the stationary node.

Given $N$ isolated mobile robots placed at unknown locations within a bounded domain $\mathcal{D} \subset \Re^{2}$, how long will it take for them to coalesce into a single connected component?

The goal of coalescence is to reach a state where all robots can communicate with each other either directly or via other robots. Clearly, coalescence time will be depend on the communication and mobility models of the robots. We use the following models.

\section{A. Communication Model}

All robots have a uniform binary disk communication with range $R$ i.e. two robots can communicate with each other if and only if the distance between them is less than $R$. We say that two robots "encounter" or "hit" each other when they are within communication range $R$ and at some time $t \geq 0$ the area that a robot has "covered" is the union of the area that has been within its communication range at $0 \leq t^{\prime} \leq t$ (figure 2 ).

\section{B. Mobility Model}

Each robot performs an independent random search for other robots. The motion strategy is a simplified version of the Random Direction model [13]. It is defined as follows (figure 2). At each time step the robot

1) chooses a direction $\theta$ uniformly in $[0,2 \pi)$

2) moves in direction $\theta$ with a constant speed $v$ for a constant distance $l$.

The random direction model results in a uniform stationary distribution.

At any point of time if two robots meet, they form a single cluster i.e. stay connected to each other and move together following the same path. Similarly when two clusters meet they coalesce to form a single cluster. Note that this interaction model does not affect the uniform stationary distribution of the clusters.

\section{Domain Model}

For ease of analysis we assume that the domain is a torus. A more realistic boundary condition is the reflecting model where the robots bounce back on hitting a boundary. However, it is known that both the torus boundary condition and reflecting boundary condition retain the stationary distribution of robots [14]. Since stationary distribution is the only condition we require of the torus boundary condition our analysis is valid for the reflecting boundary condition.

\section{Coalescence Time Analysis}

Coalescence time $(C T)$ is related to the time elapsed between robots meeting each other. Therefore to model coalescence time, we must first understand the statistics of encounter times between robots, called hitting time $(H T)$ and meeting time $(M T)$.

Definition 4.1: The hitting time of a mobility model is the time until a robot following the mobility model encounters a static robot. If robot $i$ moves according to the mobility model and robot $j$ is static, $H T=\min _{t}\left\{t:\left\|X_{i}(t)-X_{j}\right\| \leq R\right\}$.

Definition 4.2: The meeting time is the time until two robots following the same mobility model meet. If $j$ also follows the mobility model described above and starts from its stationary distribution, the meeting time between $i$ and $j$ is the time before their first encounter. $M T=\min _{t}\{t$ : $\left.\left\|X_{i}(t)-X_{j}(t)\right\| \leq R\right\}$

We will now derive the hitting time and meeting time for our mobility model.

\section{A. Hitting Time}

Let robot $i$ move according to the random mobility model and let $\alpha(t)$ represent the fraction of domain area covered by the communication disk of $i$ from time 0 to $t$. We can assume that this area is uniformly distributed in the domain. Then the new area covered in the $(t+1)^{t h}$ time step is proportional to the fraction of uncovered area in $\mathcal{D}$ and is given by

$$
\alpha(t+1)-\alpha(t) \approx(1-\alpha(t)) \frac{2 R \ell}{\mathcal{A}(\mathcal{D})}
$$

where $A(\mathcal{D})$ is the area of the domain. Solving the recursion, we get

$$
\alpha(t) \approx 1-\left(1-\frac{2 R \ell}{\mathcal{A}(\mathcal{D})}\right)^{t}
$$

If $j$ is a stationary robot placed at a random position chosen uniformly in the domain, the probability of hitting $j$ at time $t$ is equal to the fraction of the domain area covered by $i$, 


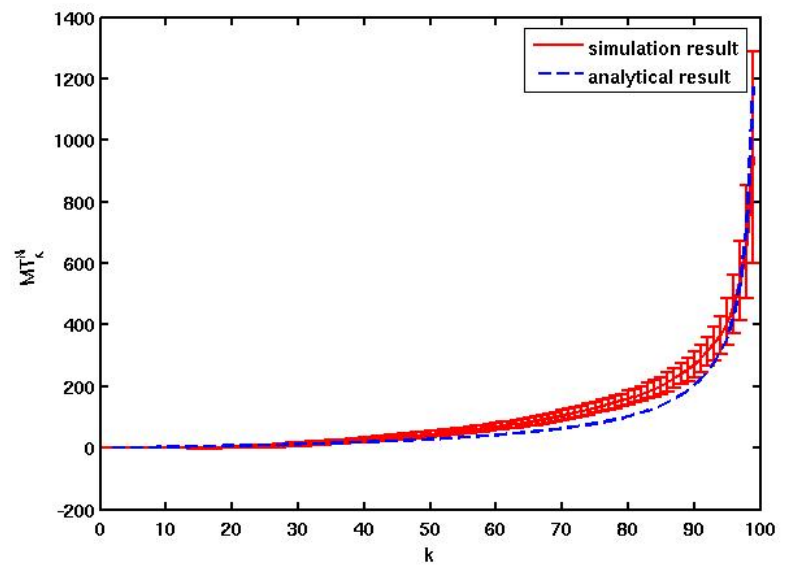

Fig. 3. Verification of analytical model for $k^{t h}$ meeting time, $M T_{k}^{N}$ derived in equation ( 9 ) for $N=100, l=100$, and $R=20$ averaged over 100 runs

$\alpha(t)$. Therefore the hitting time has the same exponential distribution as $\alpha(t)$ with mean

$$
E[H T]=\frac{\mathcal{A}(\mathcal{D})}{2 R \ell}
$$

Note that as expected, the hitting time is a function of the area of the domain, communication range, and speed (step length).

\section{B. Meeting Time}

If both $i$ and $j$ start at a random positions chosen uniformly in the domain and follow the random mobility model, then the meeting time will follow the same exponential distribution as the hitting time with mean $E[M T]=E[H T] / \hat{v}[15]$ where $\hat{v}$ is the normalized relative speed given by

$$
\hat{v}=\frac{\left\|\overrightarrow{v_{i}}-\overrightarrow{v_{j}}\right\|}{v}
$$

If $\theta$ is the angle between the velocity vectors of $i$ and $j$, then $\hat{v}$ can be computed as

$$
\frac{1}{2 \pi} \int_{0}^{2 \pi} \sqrt{(1+\cos \theta)^{2}+\sin ^{2} \theta} d \theta=1.27
$$

For $N=2$, the coalescence time $C T$ is the meeting time. $E[C T(n=2)]=E[M T]=E[H T] / 1.27$.

\section{Coalescence Time}

Let $M T_{1}^{N} \leq M T_{2}^{N} \leq \cdots \leq M T_{k}^{N} \leq \cdots \leq M T_{N-1}^{N}$ be the $1^{s t}, 2^{n d}, \cdots, k^{t h}, \cdots(N-1)^{t h}$ meeting times in a network of size $N$. The coalescence time $C T(n=N)=M T_{N-1}^{N}$.

We will now find an expression for the $k^{\text {th }}$ meeting time $M T_{k}^{N}$. The first hitting time is the minimum of $\left(\begin{array}{c}N \\ 2\end{array}\right)$ meeting times between the different pairs of robots. Since each of these is independent and has an exponential distribution,

$$
E\left[M T_{1}^{N}\right]=\frac{1}{\left(\begin{array}{c}
N \\
2
\end{array}\right)} \cdot E[M T]=\frac{2}{N(N-1)} \cdot \frac{1}{1.27} \cdot \frac{\mathcal{A}(\mathcal{D})}{2 R \ell}
$$

$t=M T_{1}^{N}$, there are $N-1$ clusters with 1 cluster consisting of two robots and $N-2$ clusters consisting of 1 robot each. At $t=M T_{k}^{N}$, there are $N-k$ clusters in the domain. The number of robots in each cluster is a random variable. At $t=M T_{k+1}^{N}$ two of these $N-k$ clusters meet and coalesce to form a bigger cluster. We expect that the time elapsed between clusters meeting will also have an exponential distribution similar to the first meeting time $M T_{1}^{N}$. The challenge is that this meeting time will depend on the size and shape of the clusters which is difficult to estimate. Even if we know the number of robots in each cluster, the shape will still be random. We will make a simplifying assumption that all clusters have the same size. Further, we assume that the expected shape of the cluster is a disc since clusters coalesce in all directions with equal probability. We validate these assumptions using simulations in MATLAB described in section V. The meeting time derived based on these assumptions is surprising close to the simulation results (figure 3 ). Based on equation (6) we write the following

$$
\begin{array}{r}
\Delta M T_{k+1}^{N}=M T_{k+1}^{N}-M T_{k}^{N} \\
E\left[\Delta M T_{k+1}^{N}\right]=\frac{2}{(N-k)(N-k-1)} \cdot \frac{1}{1.27} \cdot \frac{\mathcal{A}(\mathcal{D})}{2 R_{e f f} \ell}
\end{array}
$$

where $R_{e f f}$ is the effective radius of the clusters. $R_{e f f}$ depends not only on the size of the clusters but also the shapes. At $t=M T_{k}^{N}$, there are $N-k$ clusters. If we assume that the area of a cluster grows linearly with the number of robots the the total area of all clusters at $t=M T_{k}^{N}$ is $N \pi R^{2}-$ $k \beta \pi R^{2}$. Therefore the area of each cluster $\mathcal{C}$ at $t=M T_{k}^{N}$ is $\mathcal{A}\left[\mathcal{C}_{k}\right]=\pi R^{2}\left(\frac{N-k \beta}{N-k}\right)$ where $0 \leq \beta<1$ is a constant. For the optimal case when robots are at a distance from $R$ of each other, $\beta \approx 0.2$. Approximating the shape of each cluster as a disc, we get $R_{e f f}=\sqrt{\frac{N-k \beta}{N-k}}$. Substituting in the above equations we have

$$
\begin{array}{r}
E\left[\Delta M T_{k+1}^{N}\right] \approx \frac{2}{(N-k)(N-k-1)} \cdot \frac{E[M T]}{\sqrt{\left(\frac{N-k \beta}{N-k}\right)}} \\
E\left[M T_{k}^{N}\right]=\sum_{0}^{k-1} E\left[\Delta M T_{k+1}^{N}\right] \\
=\sum_{0}^{k-1} \frac{2}{(N-k-1) \cdot(N-k)} \frac{E[M T]}{\sqrt{\left(\frac{N-k \beta}{N-k}\right)}}
\end{array}
$$

We now have an expression for coalescence time in terms of the number of robots, $N$ and the expected meeting time $E[M T]$ which in turn is a function of size of the domain $\mathcal{D}$, communication range, $R$, and step length or speed $\ell$.

$$
\begin{aligned}
E[C T(n) & =N)]=E\left[M T_{N-1}^{N}\right]=\sum_{0}^{N-2} E\left[\Delta M T_{k+1}^{N}\right] \\
& =\sum_{0}^{N-2} \frac{2}{(N-k-1) \cdot(N-k)} \frac{E[M T]}{\sqrt{\left(\frac{N-k \beta}{N-k}\right)}}
\end{aligned}
$$


The asymptotic behavior of the coalescence time as the number of robots $N$ increases is not clear from the above expression. Therefore we will consider three cases where the analysis is tractable. In the first case, we will assume that the communication area of clusters does not change when robots join i.e. every cluster is a disk of $\pi R^{2}$ irrespective of the number robots it contains. As expected this case gives a very loose upper bound on the coalescence time. Second, we will assume that there is only one cluster that robots coalesce to upon hitting and ignore interactions between all other clusters. This case is equivalent to the stationary base station case studied in [1] and gives us a $O\left(\frac{1}{\sqrt{N}}\right)$ upper bound. Lastly, we will derive a lower bound using an idealized case where the number of clusters decreases exponentially with time i.e. at each meeting time, every cluster coalesces with exactly one other cluster.

\section{Case 1: Ignoring area growth}

At $t=M T_{k}^{N}$ there are $N-k$ clusters each of which has a disc shape of radius $R$. Substituting $R_{\text {eff }} \geq R$ in equation (8) we get

$$
\begin{array}{r}
E\left[\Delta M T_{k+1}^{N}\right] \leq \frac{2}{(N-k)(N-k-1)} \cdot E[M T] \\
E\left[M T_{N-1}^{N}\right]=\sum_{0}^{N-2} E\left[\Delta M T_{k+1}^{N}\right] \\
\leq \sum_{0}^{N-2} \frac{2}{(N-k-1) \cdot(N-k)} \cdot E[M T] \\
=\sum_{0}^{N-2} 2\left(\frac{1}{N-k-1}-\frac{1}{N-k}\right) \cdot E[M T] \\
=2(\log (2)-\log (N)+\log (N-k+1)) \cdot E[M T]
\end{array}
$$

We get, $E[C T(n=N)]=E\left[M T_{N-1}^{N}\right] \leq \log (2) \cdot M T$ i.e. the coalescence time is at most a constant times the meeting time irrespective of the size of the network. As we will see in the simulation results, this is a very loose bound for the coalescence time which means that the communication area growth plays an important role in decreasing the coalescence time.

\section{Case 2: Single cluster}

Suppose there is one special robot in the network that robots coalesce with upon meeting. Encounters between all other clusters do not affect the random motion of robots and in particular, do not result in robots coalescing. This simplifies analysis because the number of robots in different clusters is no longer random. At time $t=M T_{k}^{N}$, there is 1 cluster consisting of $k+1$ robots and $N-k-1$ single-robot clusters. This scenario was analyzed in detail in [1] where the "special robot" is called a base station. Again, we assume that the area of a cluster grows linearly with the number of robots. At $t=M T_{k}^{N}$, the cluster $C_{k}$ contains $k$ robots and $\mathcal{A}\left[\mathcal{C}_{k}\right]=\pi R^{2}(1+\beta k)$ where $0<\beta \leq 1$ is a constant. Since robots can join the cluster in all directions with equal probability, we approximate the shape of the cluster as a disk. This give us $R_{\text {eff }}=\sqrt{1+\beta \cdot k} \cdot R$. At $t=M T_{k}^{N}$, each of the $N-k-1$ isolated robots are uniformly distributed and have an equal chance of meeting the cluster. Therefore the time before the $M T_{k+1}^{N}$ is the minimum of the $N-k-1$ meeting times.

$$
\begin{array}{r}
E\left[\Delta M T_{k+1}^{N}\right] \leq \frac{1}{(N-k-1)} \cdot \frac{E[M T]}{\sqrt{1+\beta \cdot k}} \\
E\left[M T_{N-1}^{N}\right]=\sum_{0}^{N-2} E\left[\Delta M T_{k+1}^{N}\right] \\
\leq \sum_{0}^{N-2} \frac{1}{(N-k-1)} \frac{1}{\sqrt{1+\beta \cdot k}} \cdot E[M T] \\
\approx \int_{0}^{N-1}\left(\frac{1}{(N-x-1)} \frac{1}{\sqrt{1+\beta \cdot x}} \cdot E[M T]\right) d x \\
=\left(\frac{2 \cdot \tanh ^{-1}\left(\frac{\sqrt{\beta x+1}}{\sqrt{N \beta-\beta+1}}\right)}{\sqrt{N \beta-\beta+1}}\right)_{0}^{N-1} \\
=\Omega\left(\frac{1}{\sqrt{N}}\right)
\end{array}
$$

\section{Case 3: Simultaneous meeting}

We will now derive a lower bound on coalescence time by considering an extreme scenario where at each meeting time, every cluster simultaneously hits one other cluster. Let $N=2^{p}$.

$$
\begin{array}{r}
E\left[\Delta M T_{k+1}^{N}\right]=\frac{1}{\left(\begin{array}{c}
2^{p-k} \\
2
\end{array}\right)} \frac{1}{2^{k}} \cdot E[M T] \\
=\frac{1}{2^{p-k} \cdot\left(2^{p-k}-1\right)} \frac{1}{2^{k-1}} \cdot E[M T] \\
E\left[M T_{N-1}^{N}\right]=\sum_{0}^{p-1} \frac{1}{2^{p-k} \cdot\left(2^{p-k}-1\right)} \frac{1}{2^{k-1}} \cdot E[M T] \\
=2^{-p} \cdot p \cdot(\log (2)-1) \cdot E[M T] \\
=\Theta\left(\frac{\log (N)}{N}\right)
\end{array}
$$

In the next section we will verify these bounds through simulations.

\section{Simulation Results}

Simulations were conducted in MATLAB to verify the analysis. The domain used is a $2 \mathrm{D}$ torus. The parameters to the program are number of robots $N$, step size $\ell$, communication rage $R$ and torus side $T$. The robots start at random positions uniformly distributed over the torus. Every robot picks a random direction and moves with a uniform speed of 1 unit per time step. At each time step, the robot checks if it is within a distance $R$ of another robot, in which case, the robots coalesce and follow the motion of the robot with the smallest ID. After $\ell$ time steps, each cluster picks a new direction. The simulation stops when there is only one cluster. 


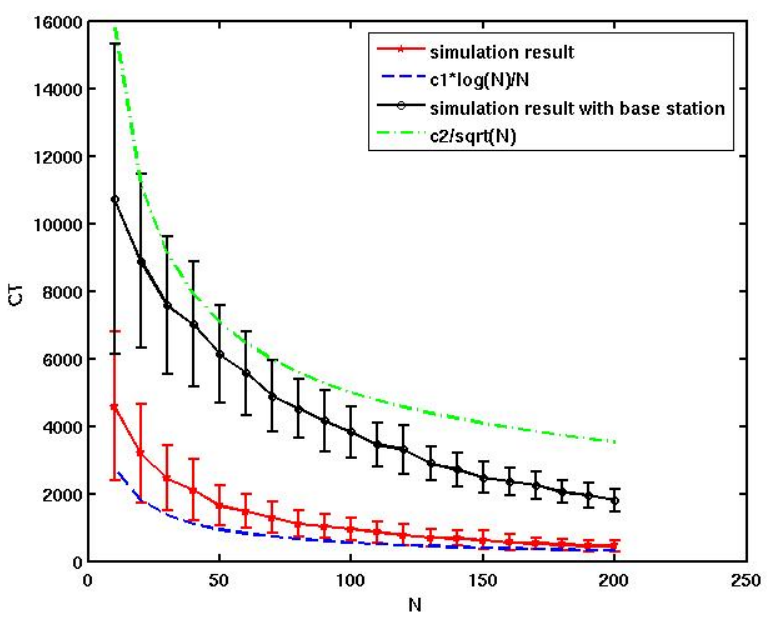

(a)

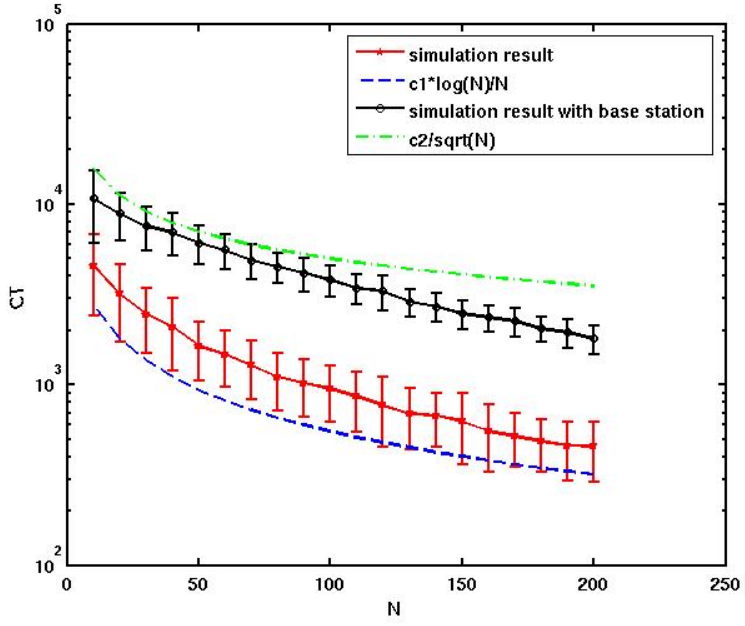

(b)

Fig. 4. The mean Coalescence Time is bounded by $O\left(\frac{1}{\sqrt{N}}\right)$ and $\Omega\left(\frac{\log (N)}{N}\right)$. The graphs shows the coalescence time $(C T)$ as a function of network size $(N)$ averaged over 200 runs with $l=100$ and $R=20$ on a (a) linear and (b) $\log$ scale.

We first verify the model for meeting time (equation (9)). This expression was was based on three simplifying assumptions 1) the clusters sizes are close to uniform, 2) the shape of the clusters is close to circular and 3) the area of a cluster grows linearly with the number of nodes. The results from simulation plotted in figure 3 are very close to the analytical expression and validate our model.

Our next set of experiments simulated Coalescence and measured Coalescence Time as a function of $N$. Figure 4 compares the mean Coalescence Time to the analytical expressions derived above. Our objective is to compare the shapes of the curves and the constants in the expression were found empirically. We see that the coalescence time has bounds of $O\left(\frac{1}{\sqrt{N}}\right)$ and $\Omega\left(\frac{\log (N)}{N}\right)$.

\section{CONCLUSION}

We consider the problem of coalescence, i.e. isolated robots independently searching for peers and forming a single connected component. Coalescence strategies can complement algorithms for robot collaboration that require a connected network. In the absence of localization or any information about the environment, the robots can perform a simple random walk search till they are within the communication range of other robots. We show using probabilistic analysis that for such a strategy, Coalescence Time has an exponential distribution which is a function of the number of robots, speed, communication range, and size of the domain. Further, it decreases as $O\left(\frac{1}{\sqrt{N}}\right)$ and $\Omega\left(\frac{\log (N)}{N}\right)$ with the number of robots, $N$. Even though the analysis is approximate, there is a surprisingly good match with the simulation results.

\section{ACKNOWLEDGMENTS}

The authors would like to thank Sundeep Pattem (University of Southern California) for helpful discussions and feedback.

\section{REFERENCES}

[1] Sameera Poduri and Gaurav S. Sukhatme, "Latency analysis of coalescence in robot groups," in IEEE International Conference on Robotics and Automation, 2007, pp. 3295-3300.

[2] J. Zhao and R. Govindan, "Understanding packet delivery performance in dense wireless sensor networks," in The First ACM Conf. on Embedded Networked Sensor Systems (Sensys'03), Nov 2003.

[3] A. Muhammad and M. Egerstedt, "On the structural complexity of multi-agent robot formations," in American Control Conference, 2004, pp. 4957- 4962.

[4] H. Ando, T. Oasa, J. Suzuki, and M. Yamashita, "Distributed memoryless point convergence algorithm for mobile robots with limited visibility," IEEE Transactions on Robotics and Automation, pp. 818828, Oct. 1999.

[5] J. Lin, A. S. Morse, and B. D. O. Anderson, "The multi-agent rendezvous problem," in International Conference on Decision and Control, 2003, pp. 1508-1513.

[6] J. Cortes, S. Martinez, and F. Bullo, "Robust rendezvous for mobile autonomous agents via proximity graphs in d dimensions," IEEE Transactions on Automatic Control, in submission.

[7] N. Roy and G. Dudek, "Collaborative exploration and rendezvous: Algorithms, performance bounds and observations," Autonomous Robots, vol. 11, no. 2, pp. 117-136, Sept. 2001.

[8] M. M. Zavlanos and G. J. Pappas, "Controlling connectivity of dynamic graphs," in International Conference on Decision and Control, 2005, pp. 6388-6393.

[9] D. Spanos and R. M. Murray, "Robust connectivity of networked vehicles," in International Conference on Decision and Control, 2004, pp. $2893-2898$.

[10] M. Porfiri, D. J. Stilwell, E. M. Bollt, and J. D. Skufca, "Random talk: Random walk and synchronizability in a moving neighborhood network," in Physica D (in press).

[11] B. Liu, P. Brass, O. Dousse, P. Nain, and D. Towsley, "Mobility improves coverage of sensor networks," in Mobihoc, 2005.

[12] P. Dykiel, "Asymptotic properties of coalescing random walks," Tech. Rep. 2005:15, Uppasala University, Dec 2005.

[13] David Aldous and James Allen Fill, "Reversible markov chains and random walks on graphs," .

[14] Christian Bettstetter, "Mobility modeling in wireless networks: categorization, smooth movement, and border effects," ACM SIGMOBILE Mobile Computing and Communications Review, vol. 5, no. 3, pp. 5566, 2001.

[15] T. Spyropoulos, K. Psounis, and C. S. Raghavendra, "Performance analysis of mobility-assisted routing.," in MobiHoc, 2006, pp. 49-60. 